\title{
DSH5, a dihydrosphingosine C4 hydroxylase gene family member, shows spatially restricted expression in rice and is lethal when expressed ectopically
}

\author{
Tomohiro Imamura ${ }^{1 \ddagger}$, Chihiro Obata ${ }^{1}$, Kazuyoshi Yoneyama $^{1}$, Masatoshi Ichikawa $^{1}$, \\ Akane Ikura ${ }^{1}$, Hiromi Mutsuro-Aoki ${ }^{1}$, Toshiki Ishikawa ${ }^{2}$, Maki Kawai-Yamada ${ }^{2}$, \\ Tadamasa Sasaki ${ }^{1 \S}$, Hiroaki Kusano ${ }^{1+\pi}$ and Hiroaki Shimada ${ }^{1 *}$ \\ ${ }^{1}$ Department of Biological Science and Technology, Tokyo University of Science, 6-3-1, Niijuku, \\ Katsushika, Tokyo 125-8585, Japan \\ ${ }^{2}$ Graduate School of Science and Engineering, Saitama University, 255 Shimo-Okubo, \\ Sakura-ku, Saitama City, Saitama 338-8570, Japan
}

(Received 5 December 2017, accepted 13 May 2018; J-STAGE Advance published date: 4 September 2018)

Dihydrosphingosine $\mathrm{C} 4$ hydroxylase (DSH), a diiron-binding membrane enzyme, catalyzes the hydration of dihydrosphingosine and acyl-sphinganine to produce phytosphingosine and phytoceramide, respectively. Rice has two types of DSH homologs: general DSHs, namely DSH1, DSH2 and DSH4, and others that show spatial expression profiles, namely DSH3 and DSH5. The general DSHs exist in many plant species. These DSHs showed similarity in their functions and complemented the yeast sur2D mutation. In contrast, homologs of DSH3 and DSH5 were found only in monocot plants. Phylogenetic analysis placed these DSHs in different clades that are evolutionarily divergent from those of the general DSHs. DSH3 and DSH5 showed low-level expression. DSH5 expression was specifically in vascular bundle tissues. Ectopic expression of DSH5 induced a dwarf phenotype characterized by severe growth inhibition and an increase in the thickness of the leaf body caused by enlargement of bulliform cells in the leaves. However, no significant difference was observed in the amount of sphingolipid species. DSH5 did not complement the yeast sur2D mutation, implying that DSH5 has little effect on sphingolipid metabolism. These findings suggested that DSH3 and DSH5 originated and diverged in monocot plants.

Key words: evolutionary divergence, gene family, monocot plant, phylogenetic analysis, phytosphingosine

\section{INTRODUCTION}

Sphingolipids, a class of lipids derived from the aliphatic amino alcohol sphingosine, are ubiquitous constituents of eukaryotic cells that also occur in some bacteria (Lynch and Dunn, 2004). The biosynthesis of sphingolipids

\footnotetext{
Edited by Kenichi Nonomura

* Corresponding author. E-mail: shimadah@rs.noda.tus.ac.jp

$\dagger$ Corresponding author. E-mail: kusano@rish.kyoto-u.ac.jp

$\ddagger$ Present Address: Research Institute for Bioresources and Biotechnology, Ishikawa Prefectural University, 1-308 Suematsu, Nonoichi, Ishikawa 921-8836, Japan

$\S$ Present Address: RIKEN Center for Sustainable Resource Science, 1-7-22, Suehiro-cho, Tsurumi-ku, Yokohama 230-0045, Japan

II Present Address: Laboratory of Plant Gene Expression, Research Institute of Sustainable Humanosphere, Kyoto University, Gokasho, Uji 611-0011, Japan

DOI: http://doi.org/10.1266/ggs.17-00054
}

starts with the condensation of L-serine and palmitoylCoA to form 3-ketosphinganine (Tamura et al., 2001), which is then converted to dihydrosphingosine (DHS) by NADPH-dependent reduction at the C3 position (Beeler et al., 1998).

DHS and its derivatives are composed of sphingoid long-chain bases (LCBs) (Lynch and Dunn, 2004). The composition of LCBs is divided into three main types: mammalian, yeast and plant (Dickson, 1998; Lynch and Dunn, 2004). In mammals, sphingolipids mainly occur as ceramide derived from the $\mathrm{N}$-acylation of DHS; these sphingolipids have both a long-chain fatty acid, ranging from 14 to 26 carbons in length, and a double bond at the C4-C5 position (Michel et al., 1997). In yeast, most DHS is hydroxylated at the $\mathrm{C} 4$ position and converted to 4-hydroxysphinganine, which is also known as phytosphingosine (PHS) (Dickson, 1998; Wright et al., 2003). In plants, a proportion of PHS is further modified by desatu- 
ration at the $\mathrm{C} 8-\mathrm{C} 9$ position following PHS synthesis, as occurs in yeast (Sperling et al., 1998). Plant ceramide (phytoceramide) is composed of these compounds, as is the mammalian-type ceramide (Lynch and Dunn, 2004).

Dihydrosphingosine $\mathrm{C} 4$ hydroxylase (DSH) catalyzes the hydration of the $\mathrm{C} 4$ position of DHS and acyl-sphinganine to produce PHS and phytoceramide, respectively (Haak et al., 1997; Grilley et al., 1998). DSHs are diiron-binding membrane enzymes involved in oxygen-dependent modifications of hydrocarbon substrates, and have a highly conserved structure among plant species (Grilley et al., 1998; Sperling et al., 2001; Imamura et al., 2007).

The activity of DSH is believed to define the DHS/ PHS balance of sphingoid bases in yeast and of plant sphingolipids (Haak et al., 1997; Sperling and Heinz, 2003). DSH is considered a key enzyme in PHS synthesis; however, DSH (SUR2) activity, which is required for the hydroxylation of C-4 of the sphingoid moiety of ceramide, is not essential for the growth of Saccharomyces cerevisiae (Haak et al., 1997; Grilley et al., 1998). Two Arabidopsis thaliana DSH genes, SBH1 and SBH2, have been reported, and they complement yeast SUR2 gene function (Sperling et al., 2001). In Arabidopsis, the synthesis of trihydroxy LCBs plays a central role in maintaining growth and mediating the total content and fatty acid composition of sphingolipids. Double mutants, which completely lacked trihydroxy LCBs, were severely dwarfed, did not progress from vegetative to reproductive growth, and had enhanced expression of programmed cell death-associated genes. In these mutants, increases in sphingolipid content resulted from the accumulation of molecular species that have C16 fatty acids rather than very-long-chain fatty acids (VLCFAs) (Chen et al., 2008).

The importance of $S B H$ genes in Arabidopsis growth can be explained by the plant's requirement for the ceramide species and by the substrate specificity of ceramide synthases. The Arabidopsis ceramide synthases LOH1 and LOH3 but not LOH2 are essential for plant growth (Markham et al., 2011; Ternes et al., 2011). The knockdown loh1-1 loh3-1 double mutant shows a less drastic reduction in VLCFA-containing sphingolipids and reduced growth. loh2 mutant seedlings show both a strong reduction of 16:0 fatty acids in ceramide and other sphingolipids and a significant increase in VLCFA-containing sphingolipids. These results suggest the existence of two classes of ceramide synthases that have different substrate specificities, one preferring both $\mathrm{C} 16$ fatty acids and dihydroxy-LCBs and the other preferring both VLCFAs and trihydroxy LCBs (Markham et al., 2013). In contrast, no phenotype is detected in mutant yeast cells with a deletion of either $L A G 1$ or $L A C 1 / D G T 1$, which are homologs of the $L O H$ genes, whereas lag1 $1 \Delta$ dgt1 $\Delta$ cells display growth defects (Barz and Walter, 1999), suggesting functional redundancy. The unique evolutionary differentiation of plant
$L O H$ genes may provide roles for $D S H$ genes in the control of the ceramide biosynthesis pathway in plants.

The rice genome contains five homologous DSH genes, DSH1-DSH5, whose gene products show sequence similarity to yeast SUR2. We have found that DSH1 and DSH4 complement the yeast sur2D mutation. We have also reported that $D S H 1$ is essential for fertility even though rice has five similar $D S H$ genes in its genome. RNAi-mediated knockdown of DSH1 stimulated the up-regulation of $\mathrm{DSH} 2$ in vegetative organs but not in reproductive organs (Imamura et al., 2007). DSH3 and DSH5 are diverged from other DSH family members, having relatively low sequence conservation with the others. These observations suggest that there is functional conservation but physiological differentiation among the members of the rice $D S H$ gene family. Here, we report functional differences among rice DSHs and discuss the evolutionary divergence within the $D S H$ gene family in plants.

\section{MATERIALS AND METHODS}

Plant materials and growth conditions Oryza sativa L. cv. Nipponbare was used as the wild-type plant. Seeds were germinated at $30^{\circ} \mathrm{C}$ in a dark chamber. The seedlings were grown under continuous light $\left(13 \mu \mathrm{mol} \mathrm{m} \mathrm{m}^{-2}\right.$ $\mathrm{s}^{-1}$ ) for three days. The rice plants were cultivated in a growth chamber with $80 \%$ humidity under long-day conditions that consisted of a cycle of $14 \mathrm{~h}$ of light $\left(30^{\circ} \mathrm{C}\right.$, $270 \mu \mathrm{mol} \mathrm{m}{ }^{-2} \mathrm{~s}^{-1}$ ) and $10 \mathrm{~h}$ of dark at $25^{\circ} \mathrm{C}$.

RNA quantification using real-time RT-PCR Realtime RT-PCR (reverse transcription-PCR) was carried out according to previously described methods (Imamura et al., 2007). The amounts of DSH1 (Os06g0227200), DSH2 (Os02g0745700), DSH4 (Os07g0627700) and DSH5 (Os01g0658600) transcripts were determined as values relative to those of the Actin1 transcript (acc. no. X16280). Detailed procedures are given in the Supplemental Methods.

Reporter assays using the $\beta$-glucuronidase (GUS) gene The predicted $1.2 \mathrm{~kb}$ promoter region of $\mathrm{DSH} 5$ was prepared from rice genomic DNA using PCR with the primers 5'-AAGAATTCATAGTATAGATAGAATGGCTGGAT-3' and 5'-TACCATGGTTGCAACTAGAAAGCTAGTGCTA-3' and inserted into the region preceding the GUS ORF in pCAMBIA1381 as described previously (Imamura et al., 2007). The resultant plasmid was used for transformation of rice plants using the Agrobacteriummediated method (Hiei et al., 1994). GUS activity was detected by staining using a reaction buffer containing $1 \mathrm{mM}$ X-Gluc (5-bromo-4-chloro-3-indolyl- $\beta$-glucuronide), $50 \mathrm{mM}$ sodium phosphate buffer ( $\mathrm{pH} 7.0), 5 \%(\mathrm{v} / \mathrm{v})$ methanol and $0.1 \%(\mathrm{v} / \mathrm{v})$ Triton X-100 after incubation for $16 \mathrm{~h}$ 
according to the methods of Jefferson et al. (1987).

Creation of the gene for DSH5 transformants For the forced expression of $\mathrm{DSH} 5$, the coding region was inserted into the region following the CaMV 35S promoter in pGWB2 (Nakagawa et al., 2007). For the ectopic expression of $\mathrm{DSH} 5$, the region for the DSH5 ORF was prepared by PCR from the corresponding cDNA using the primers 5'-CACCATGGGGTTCATCAGCGGCGAGGAGC-3' and 5'-CAACGATCGCCACGTTCTCCTG-3', and then inserted into pENTR-D-TOPO. The resultant plasmid was used for construction of the appropriate plasmids by LR clonase using pGWB2 (Nakagawa et al., 2007).

\section{Analysis of LCBs and sphingolipid composition} Yeast sur2D mutants containing the expression plasmid for each of $D S H 1, D S H 2, D S H 4$ or $D S H 5$ were prepared using a pYES2 vector. The LCBs in the transformants were analyzed using HPLC as described previously (Imamura et al., 2007). The sphingolipid composition in plant leaves was analyzed according to the methods of Ishikawa et al. (2016). Leaves were freeze-dried prior to the analysis.

Complementation test in yeast Complementation by DSH1, DSH2, DSH4 and DSH5 of the yeast sur2D mutation was determined as described previously (Imamura et al., 2007). The cultured cell suspension was diluted with YNB medium (Becton Dickinson Japan, Tokyo) to adjust its concentration to $\mathrm{OD}_{600}=0.3$, and then portioned to a series of 1:2 dilutions ranging from 1:2 to 1:32. Each aliquot was inoculated onto YNB plates, in which $4 \mu \mathrm{g} / \mathrm{ml}$ syringomycin E was supplemented as required. Cells were grown at $30{ }^{\circ} \mathrm{C}$.

Phylogenetic analysis Homologs of DSH1, DSH2, DSH3, DSH4 and DSH5 in various plant species were queried using the BLAST program and the DDBJ (http:// www.ddbj.nig.ac.jp/). The amino acid sequences were aligned using MUSCLE (Edgar, 2004), and the maximum likelihood tree was constructed using MEGA7 (Kumar et al., 2016) based on the JTT matrix-based model (Jones et al., 1992) with 1,000 bootstrap replicates.

\section{RESULTS}

Phylogenetic analysis of DSHs There are five $D S H$ genes in the rice genome: $\mathrm{DSH} 1$ to $\mathrm{DSH}$. Homologs of DSHs are widely distributed in the plant kingdom. We constructed a phylogenetic tree of the DSHs of various plant species including a moss (Physcomitrella patens) and a fern (Selaginella moellendorffii). The tree showed that the homologs could be divided into two groups based on their evolutionary relationship; one group had subfamilies containing homologs of DSH1, DSH2 and DSH4,
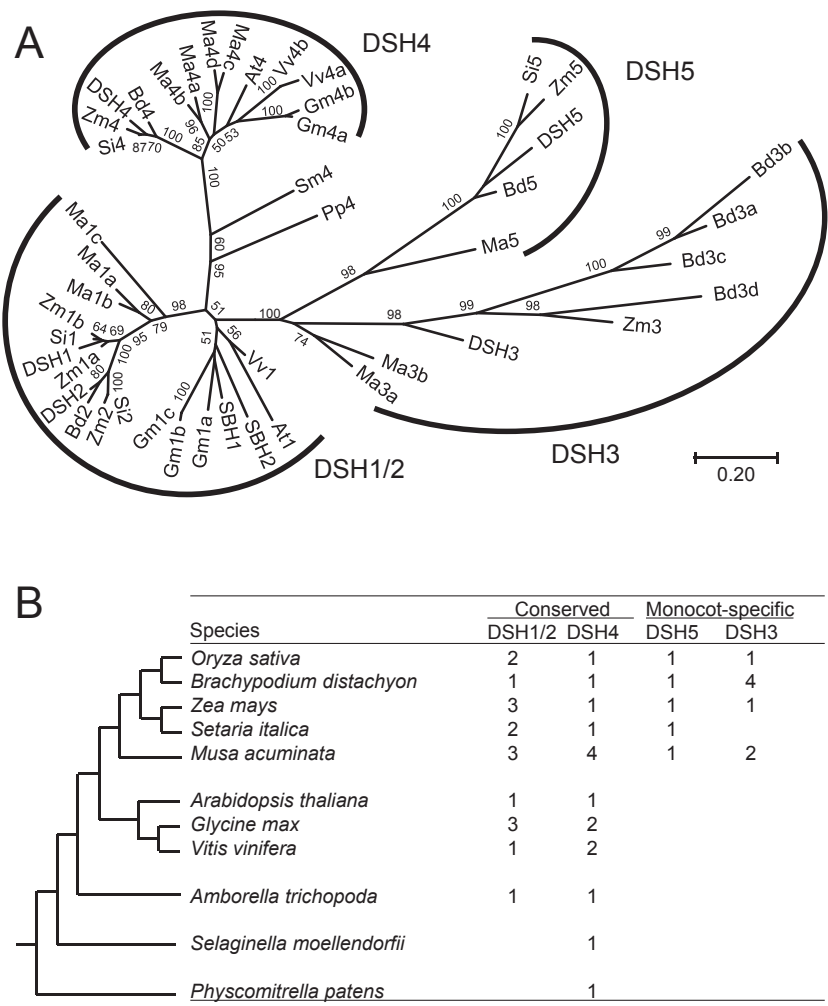

Fig. 1. Phylogenetic tree of DSHs. (A) Phylogenetic analysis of rice DSHs (DSH1 - DSH5) and their homologs in other plant species: Brachypodium distachyon (Bd), Zea mays (Zm), Setaria italica (Si), Musa acuminata (Ma), Arabidopsis thaliana (SBH), Glycine $\max (\mathrm{Gm})$, Vitis vinifera (Vv), Amborella trichopoda (At), Selaginella moellendorffii (Sm), Physcomitrella patens $(\mathrm{Pp})$. Proteins are shown as the names of plant species with the corresponding accession numbers registered in protein databases (http://www.ncbi.nih.gov/ and http://www.ddbj.nig.ac.jp/ index-j.html). Accession numbers of individual proteins are as follows: DSH1, NP_917342.1; DSH2, NP_001048112; DSH3, NP_001057540; DSH4， NP_001060346; DSH5， BAB85258; Arabidopsis SBH1, AAL50102; Arabidopsis SBH2, AAN64519; Zm1a, NP_001149259; Zm1b, ACG37950; Zm2, NP_001149772; Zm3, ACF85550; Zm4, ACG26719; Zm5, NP_001309955; Bd2, XP_003570655; Bd3a，XP_003576210; Bd3b，XP_003573123; Bd3c, XP_003561729; Bd3d, XP_003561259; Bd4, XP_003562637; Bd5, XP_003566953; Si1, XP_004965037; Si2, XP_004953861; Si4, XP_004958394;Si5XP_004972049;Ma1a,XP_009390920.1; Ma1b, XP_009412677.1;Ma1c,XP_009409998.1; Ma3a,XP_009405035.1; Ma3b, XP_009398168.1; Ma4a, XP_009404416.1; Ma4b, XP_009389566.1;Ma4c,XP_009410375.1;Ma4d,XP_009395920.1; Ma5, XP_009410862.1; Gm1a, AK245909; Gm1b, XP_003517969; Gm1c, XP_003518724; Gm4a, NP_001242157; Gm4b, NP_001242851; Vv1, XP_002275229; Vv4a, XP_003634932; Vv4b, XP_002265496; At1，XP_006827207.1; At4，XP_006827130.1; Sm4, XP_002967466; and Pp4, XP_001777484. The subfamilies DSH1/2, DSH3, DSH4 and DSH5 are indicated in the figure. Numbers represent bootstrap values. The bar represents the number of substitutions per site. (B) Variation in the gene numbers of DSH homologs of 11 representative plant species. The gene numbers classified in each subfamily group are tallied. The topology of species evolution is drawn according to APG IV (The Angiosperm Phylogeny Group, 2016). 
and the other contained those of DSH3 and DSH5 (Fig. 1A). Homologs of DSH1, DSH2 and DSH4 exist in many plant species. However, homologs of DSH3 and DSH5 were found only in monocot plants (Fig. 1B). This suggested that they originated after the divergence of monocot plants in angiosperms.

Expression patterns of rice $D S H$ genes Among the $D S H$ genes, we have determined that $D S H 1$ (Os06g0227200), DSH2 (Os02g0745700) and DSH4 (Os07g0627700) are abundantly expressed, but we have not detected any DSH3 (Os06g0330400) transcripts (Imamura et al., 2007). To date, the rice expression database RiceXPro (http://ricexpro.dna.affrc.go.jp/) has shown that $D S H 3$ is specifically but weakly expressed both in the roots during vegetative stages and in the pistil, whereas $D S H 1, D S H 2$ and $D S H 4$ are highly expressed in many organs (Supplementary Fig. S1). However, there are no registered cDNA data and no reports on the expression profile for DSH5 (Os01g0658600) in the database.

In this work, we detected DSH5 transcription. Realtime RT-PCR detected transcripts of the DSH5 gene specifically in the leaves (Fig. 2). The spatial pattern of DSH5 gene expression was analyzed using a GUS reporter gene. GUS activity was detected in the cells of vascular bundle tissues in the leaves, roots, glumes and filaments of the stamen (Fig. 3).

To determine functional similarity between rice DSH5 and other DSHs, we transformed the yeast sur2D mutant with rice $D S H 1, D S H 2, D S H 4$ and $D S H 5$ cDNAs. We detected phytosphingosine (PHS) and dihydrosphingosine (DHS) in the transformants of the sur2D mutant in which either $\mathrm{DSH} 2$ or $\mathrm{DSH} 4$ was expressed. However, No PHS was produced in the yeast transformant containing $\mathrm{DSH} 5$ (Fig. 4A). The PHS/DHS ratio was higher in yeast cells containing $\mathrm{DSH} 4$ than those containing $\mathrm{DSH} 1$ and $\mathrm{DSH} 2$ (Fig. 4B). The phenotype of the yeast sur2D mutation can be determined by the acquisition of tolerance to syringomycin E (Grilly et al., 1998). As shown in Fig. $4 \mathrm{C}$, the sur $2 \mathrm{D}$ mutant containing $\mathrm{DSH} 4$ lacked tolerance to syringomycin $\mathrm{E}$, and that containing $D S H 1$ was less tolerant than the sur $2 D$ mutant. However, the mutant containing DSH5 maintained obvious tolerance to syringomycin $\mathrm{E}$, indicating that DHS5 did not complement the sur $2 D$ mutation. These results suggest that $D S H 5$ has a different function from $\mathrm{DSH} 1, \mathrm{DSH} 2$ and $\mathrm{DSH} 4$.

Ectopic expression of DSH5 leads to a lethal phenotype DSH5 shows relatively low similarity to the other DSHs and has differences at many amino acid positions, although it possesses highly conserved histidine residues within the diiron center region (data not shown). To understand the functional difference between DSH5 and other DSHs, we created transformed rice plants (DSH5ox) that contained the DSH5 gene, whose expression was
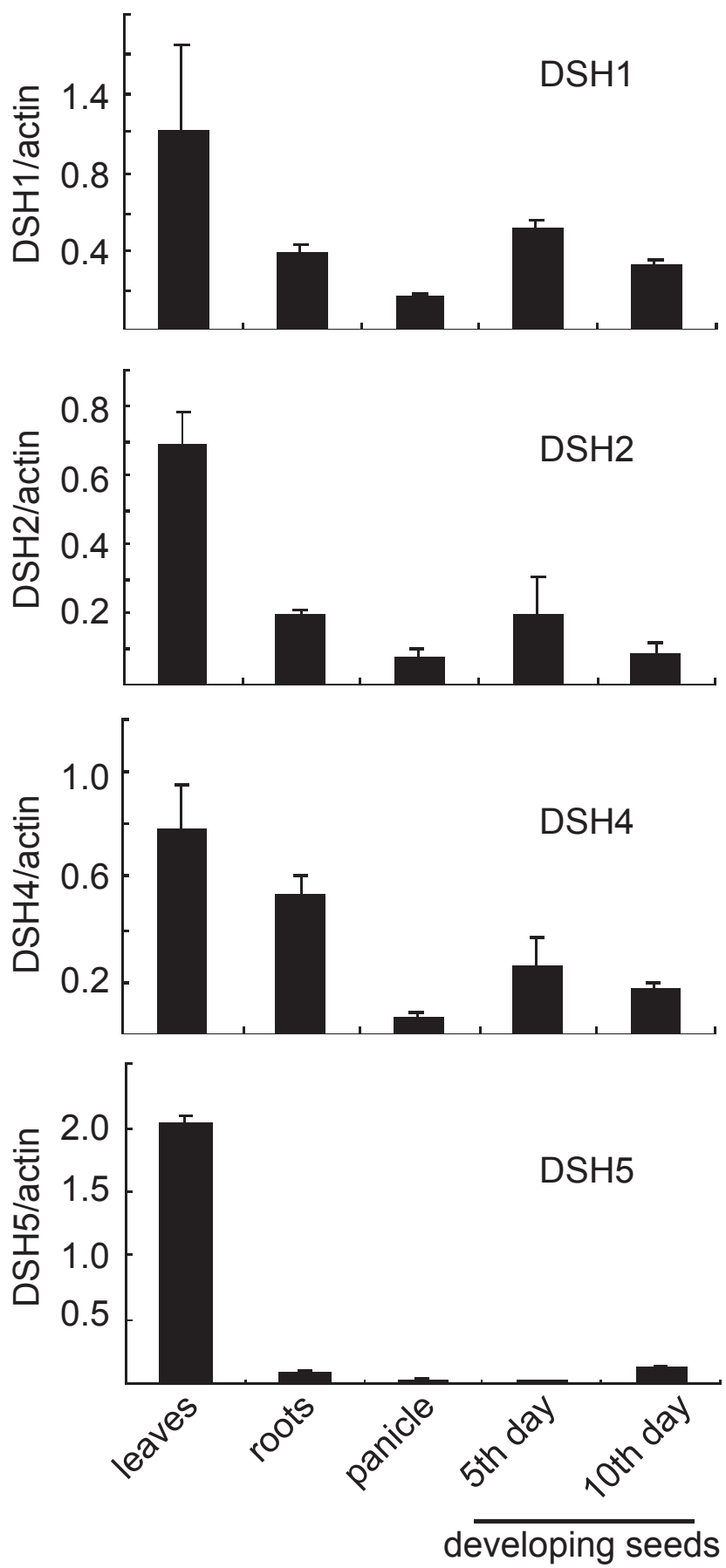

Fig. 2. Expression of rice $D S H$ genes. Real-time RT-PCR analysis of the expression of rice $D S H$ genes. Total RNA was prepared from leaves, roots and panicles, and from developing seeds at 5 days and 10 days after flowering. Values relative to those of Actin1 (actin) are indicated. Error bars represent standard deviation $(\mathrm{N}=3)$.

driven by the CaMV $35 \mathrm{~S}$ promoter.

We obtained many DSH5-overexpressing transformants, but all of them showed a dwarf phenotype and their mature leaves were small. From the DSH5transformant callus, we regenerated numerous plants, 

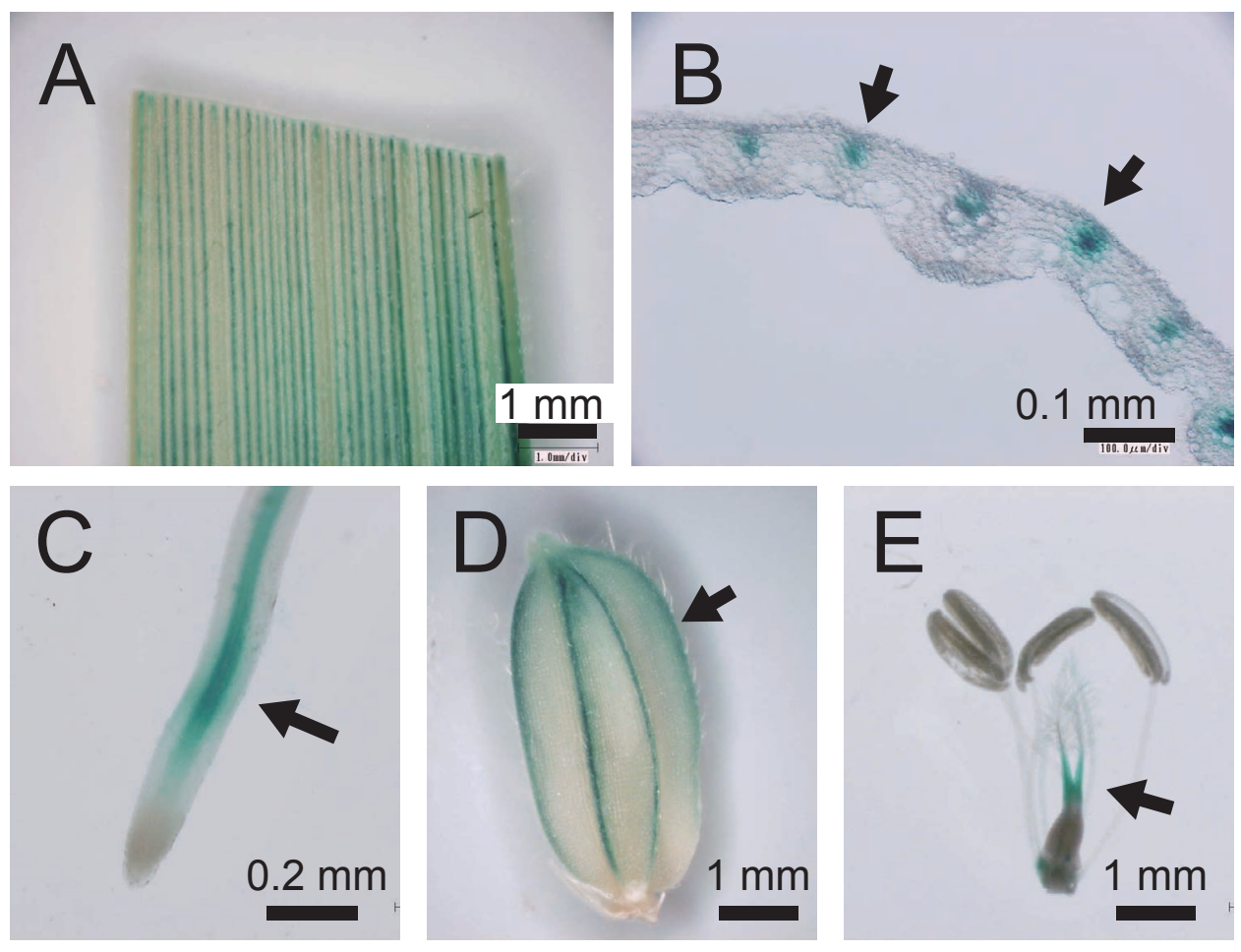

Fig. 3. Histochemical analysis of GUS representing the expression of DSH5. Microscopic observations of a leaf (A), a cross-section of a leaf (B), a root (C), a spikelet (D) and a spikelet without glumes (E) are shown. Arrows indicate stained regions.

which were transplanted to pots for culture in a greenhouse when they reached a height of $\sim 10 \mathrm{~cm}$. However, the growth of these plants was severely reduced. Most of them stopped gaining height, and died during the vegetative stage. Plant height of the DSH5 transformant was severely reduced (Fig. 5A-5C). We observed that bulliform cells in the leaves were significantly enlarged, resulting in an increased thickness of the leaf body; however, no morphological change was observed in the vascular bundles in the leaves (Fig. 5D-5F).

We analyzed the amounts of representative species of sphingolipids in the transformants. However, no marked differences in the amounts of these species, or the ratio of the t18/d18 species, were detected between DSH5ox and WT plants (Fig. 5G). These results suggest that ectopic DSH5 expression had little effect on sphingolipid metabolism, even though significant morphological changes were observed.

\section{DISCUSSION}

DSH1, DSH2 and DSH4 showed functional similarity to each other. Yeast transformants containing these genes exhibited PHS synthesis activity. These DSHs complemented the yeast sur2D mutation. In contrast, DSH5 did not produce any PHS in the yeast sur2D mutant, and the yeast $\operatorname{sur} 2 D$ mutation was not complemented by the introduction of DSH5 (Fig. 4).

In our previous work, we did not detect any transcripts of DSH3 or DSH5 (Imamura et al., 2007). Currently, the established database RiceXPro indicates that $\mathrm{DSH} 3$ is specifically expressed in roots and pistils (Supplementary Fig. S1). However, there is no description of DSH5 gene expression in the database. In the present work, we detected transcripts of rice DSH5. Its expression pattern was highly specific to vascular bundle tissues (Fig. 3). Ectopic expression of DSH5 in transgenic rice plants resulted in severe growth inhibition in the vegetative stage, and these plants did not survive to the reproductive stage (Fig. 5); however, no evident alteration in the composition of sphingolipids occurred in the DSH5 transformants (Fig. 5). Therefore, we conclude that DSH5 has some unknown function that largely influences plant growth. These results imply that the DSH5 protein is active at the specific location where it is expressed, although its function is unclear.

DSH3 and DSH5 exhibit relatively low similarity with the general DSHs. Homologs of DSH3 and DSH5 are widespread in monocot plant species but were not found in any dicot species (Fig. 1). These homologs composed two different clades that had evolutionarily diverged. Brachypodium distachyon and Musa acuminata have multiple DSH3 homologs, but Zea mays and rice possess only a single counterpart; only one DSH5 

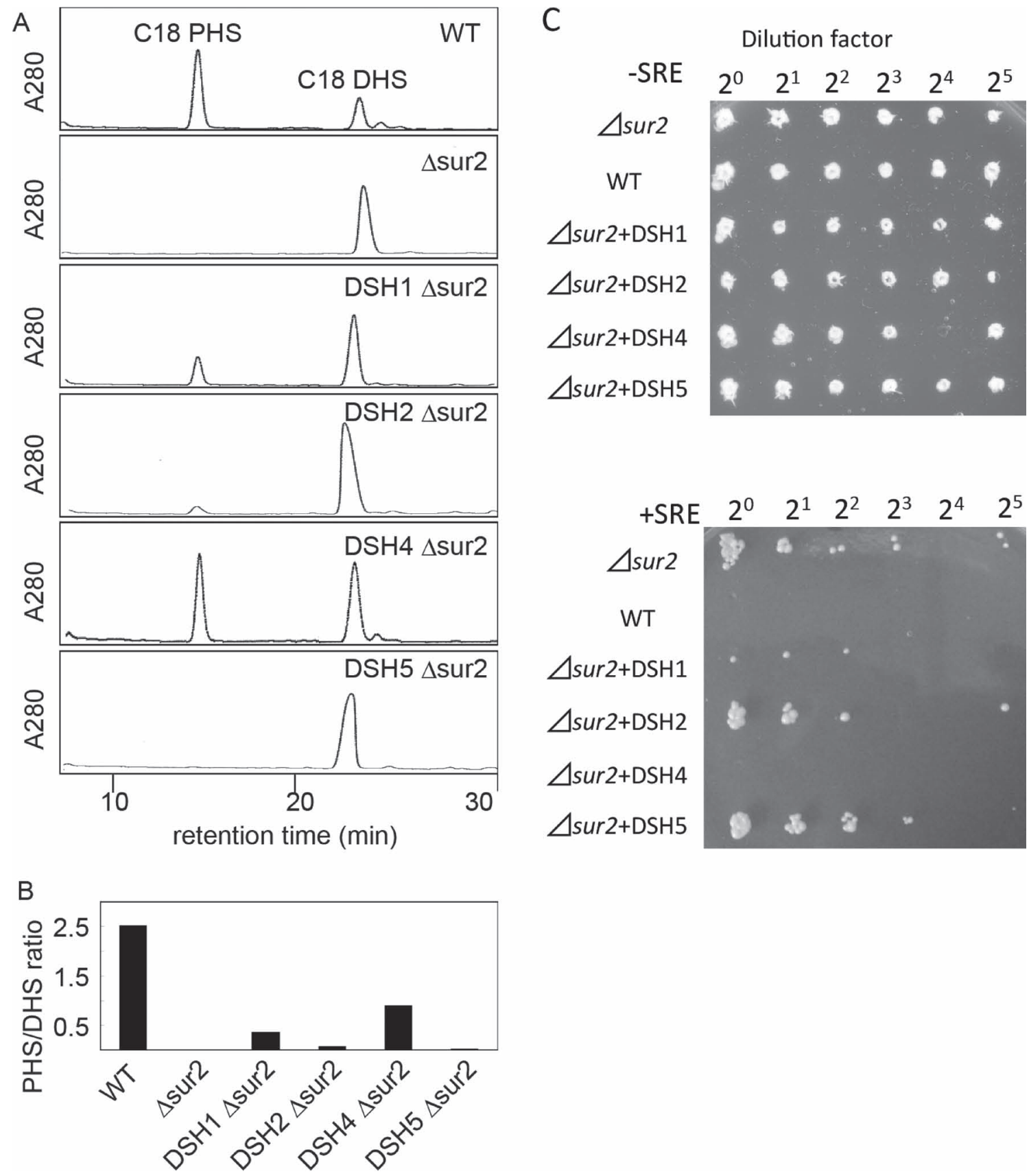

Fig. 4. Complementation test using the yeast sur2 mutant. (A) Chromatograms of the HPLC results of C18-phytosphingosine (PHS) and C18-dihydrosphingosine (DHS) analysis in transgenic yeast. $\Delta$ sur2, sur2D mutant; DSH1 $\Delta$ sur2, sur2D mutant harboring the DSH1 gene; DSH2 $\Delta$ sur2, sur2D mutant harboring the DSH2 gene; DSH4 $\Delta$ sur2, sur2D mutant harboring the DSH4 gene; DSH5 $\Delta$ sur2, sur2D mutant harboring the DSH5 gene; WT, wild-type yeast. (B) Ratio of the values of the integrated signal for PHS to that of DHS. The values were calculated using the chromatograms. (C) Tolerance to syringomycin $\mathrm{E}$ in yeast sur2D mutants. sur2D mutants harboring $D S H 1, D S H 2, D S H 4$ or $D S H 5$, and WT, were grown on a YNB agar plate or a YNB agar plate containing $4 \mu \mathrm{g} / \mathrm{ml}$ syringomycin E. A series of cell dilutions were inoculated onto the plate. -SRE and + SRE, absence and presence of syringomycin E in the medium, respectively.

homolog was found in the monocot plant species (Fig. 1). These homologs probably originated from the general DSHs and have become highly divergent in monocot plants. We propose the following history of DSH evolution in plants: an ancestral DSH enzyme originated in lower plants. DSH homologs were generated by duplication and dispersed in angiosperms. These homologs were established as general DSHs but then diverged into two major groups, one containing DSH1 and DSH2 and the other containing DSH4. Monocot plants acquired 

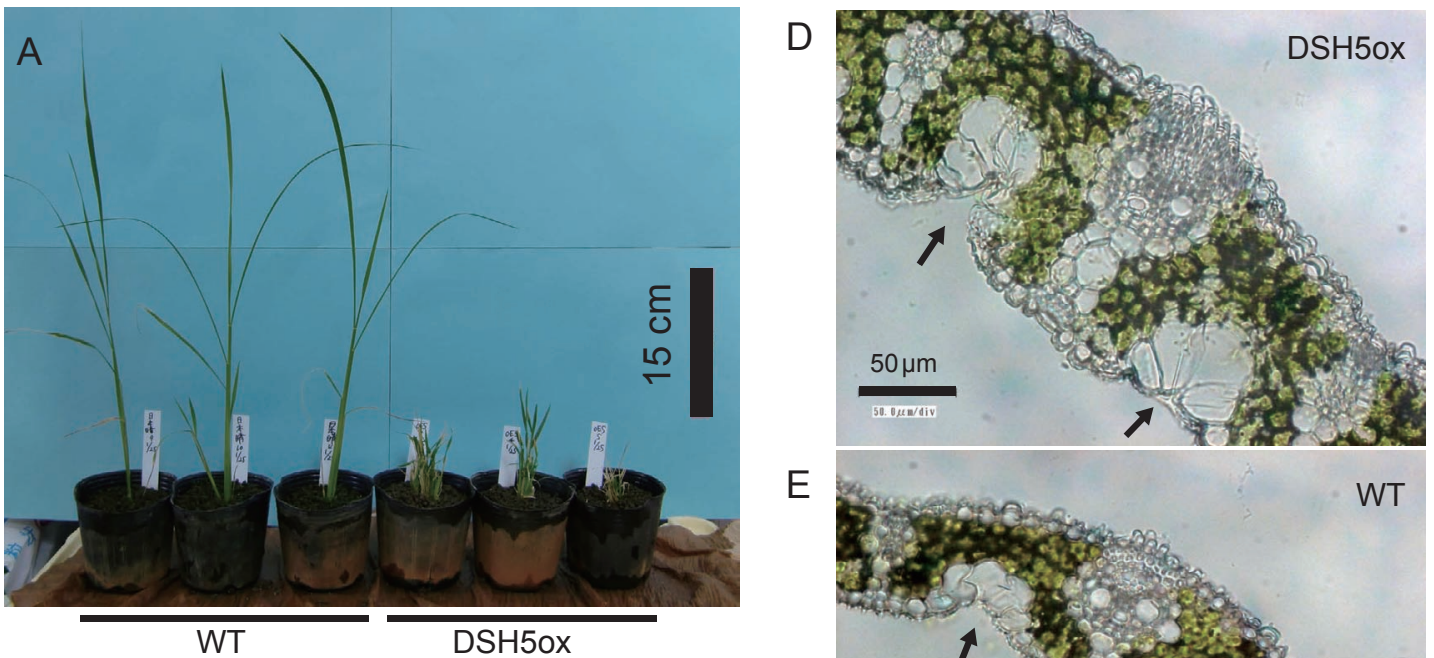

$\mathrm{E}$
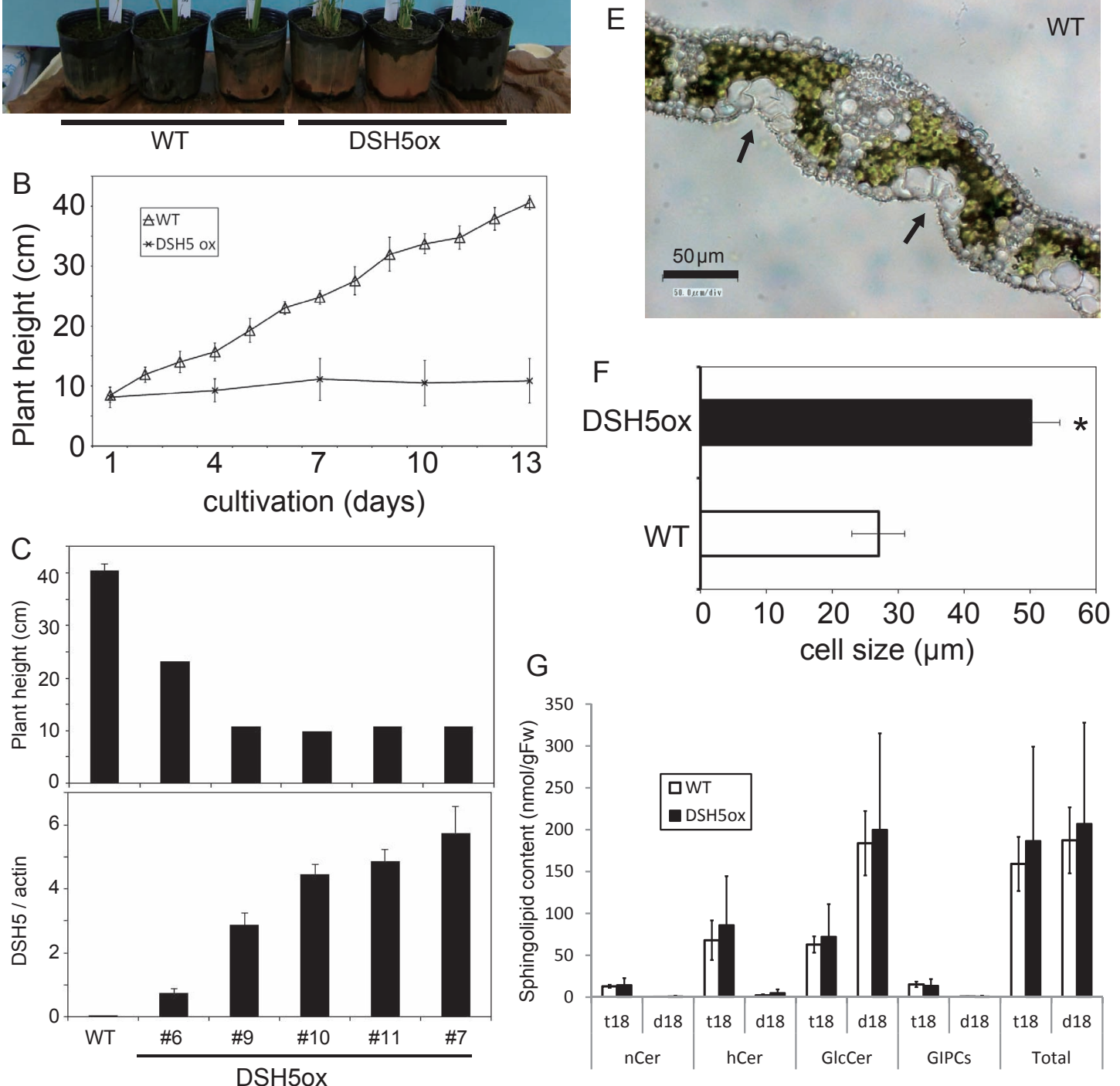

Fig. 5. Morphology of rice transformants harboring the gene for ectopic expression of DSH5. (A) Comparison of the DSH5-overexpressing (DSH5ox) transformants and wild-type (WT) rice plants. The picture shows features of 2-week-old plants. (B) Time course of the growth of DSH5ox transformant plants compared with that of WT control plants. Error bars represent the standard error for six independent plants. (C) Height of 2-week-old plants, compared with the abundance of DSH5 transcripts in the corresponding plants; transcript expresison values are relative to Actin1 (actin) transcripts. Six independent transgenic plants were analyzed. Numbers with the prefix "\#” indicate individual DSH5ox transformants. Error bars represent means \pm SEs. (D, E) Cross-sections of a leaf of a DSH5ox transformant and a WT control plant. Arrows indicate bulliform cells. (F) Size of bulliform cells. Width of bulliform cells was measured in the microscopic images. Values shown are means \pm SEs of four plants. $* P<0.05$. (G) Sphingolipidome study of the ectopic expression of DSH5. Sphingolipid species in the DSH5 transformants (DSH5ox) were compared with those of control plants (WT). The trihydroxy (t18) and dihydroxy (d18) sphingolipid content of ceramide with non-hydroxy fatty acid (nCer), ceramide with 2-hydroxy fatty acid (hCer), glycosyl ceramide (GlcCer), and glycosyl inositol phosphoceramides (GIPCs) was determined. Error bars represent standard deviation $(\mathrm{N}=4)$. 
a second type of DSH homolog, comprising DSH3 and DSH5, which may have specialized roles, as they show distinctive expression patterns in specific regions of the plant body, namely the roots and bundle tissues in the leaves, respectively. Ectopic expression of DSH5 had lethal effects on plant growth (Fig. 5), which suggests that DSH5 homologs have unique characters that are different from those of the general DSHs.

We thank Kao-Chih She and Kanako Sensui for technical assistance and thank Tomoyuki Furukawa for valuable suggestions. Syringomycin E was kindly supplied by J. Takemoto at Utah State University.

\section{REFERENCES}

Barz, W. P., and Walter, P. (1999) Two endoplasmic reticulum (ER) membrane proteins that facilitate ER-to-Golgi transport of glycosylphosphatidylinositol-anchored proteins. Mol. Biol. Cell. 10, 1043-1059.

Beeler, T., Bacikova, D., Gable, K., Hopkins, L., Johnson, C., Slife, H., and Dunn, T. (1998) The Saccharomyces cerevisiae TSC10/YBR265w gene encoding 3-ketosphinganine reductase is identified in a screen for temperature-sensitive suppression of the $\mathrm{Ca}^{2+}$-sensitive $\operatorname{csg} 2 \Delta$ mutant. J. Biol. Chem. 273, 30688-30694.

Chen, M., Markham, J. E., Dietrich, C. R., Jaworski, J. G., and Cahoon, E. B. (2008) Sphingolipid long-chain base hydroxylation is important for growth and regulation of sphingolipid content and composition in Arabidopsis. Plant Cell 20, 1862-1878.

Dickson. R. C. (1998) Sphingolipid functions in Saccharomyces cerevisiae: comparison to mammals. Annu. Rev. Biochem. 67, 27-48.

Edgar, R. C. (2004) MUSCLE: multiple sequence alignment with high accuracy and high throughput. Nucleic Acids Res. 32, 1792-1797.

Grilley, M. M., Stock, S. D., Dickson, R. C., Lester, R. L., and Takemoto, J. Y. (1998) Syringomycin action gene SYR2 is essential for sphingolipid 4-hydroxylation in Saccharomyces cerevisiae. J. Biol. Chem. 273, 11062-11068.

Haak, D., Gable, K., Beeler, T., and Dunn, T. (1997) Hydroxylation of Saccharomyces cerevisiae ceramides requires Sur2p and Scs7p. J. Biol. Chem. 272, 29704-29710.

Hiei, Y., Ohta, S., Komari, T., and Kumashiro, T. (1994) Efficient transformation of rice (Oryza sativa L.) mediated by Agrobacterium and sequence analysis of the boundaries of the T-DNA. Plant J. 6, 271-282.

Imamura, T., Kusano, H., Kajigaya, Y., Ichikawa, M., and Shimada, H. (2007) A rice dihydrosphingosine C4 hydroxylase (DSH1) gene, which is abundantly expressed in the stigmas, vascular cells and apical meristem, may be involved in fertility. Plant Cell Physiol. 48, 1108-1120.

Ishikawa, T., Ito, Y., and Kawai-Yamada, M. (2016) Molecular characterization and targeted quantitative profiling of the sphingolipidome in rice. Plant J. 88, 681-693.

Jefferson, R. A., Kavanagh, T. A., and Bevan, M. W. (1987) GUS fusions: $\beta$-glucuronidase as a sensitive and versatile gene fusion marker in higher plants. EMBO J. 6, 3901-3907.

Jones, D. T., Taylor, W. R., and Thornton, J. M. (1992) The rapid feneration of mutation data matrices from protein sequences. Comput. Appl. Biosci. 8, 275-282.

Kumar, S., Stecher, G., and Tamura, K. (2016) MEGA7: molecular evolutionary genetics analysis version 7.0. Mol. Biol. Evol. 33, 1870-1874.

Lynch, D. V., and Dunn, T. M. (2004) An introduction to plant sphingolipids and a review of recent advances in understanding their metabolism and function. New Phytol. 161, 677-702.

Markham, J. E., Lynch, D. V., Napier, J. A., Dunn, T. M., and Cahoon, E. B. (2013) Plant sphingolipids: function follows form. Curr. Opin. Plant Biol. 16, 350-357.

Markham, J. E., Molino, D., Gissot, L., Bellec, Y., Hématy, K., Marion, J., Belcram, K., Palauqui, J. C., Satiat-JeuneMaître, B., and Faure, J. D. (2011) Sphingolipids containing verylong-chain fatty acids define a secretory pathway for specific polar plasma membrane protein targeting in Arabidopsis. Plant Cell 23, 2362-2378.

Michel, C., van Echten-Deckert, G., Rother, J., Sandhoff, K., Wang, E., and Merrill, A. H. Jr. (1997) Characterization of ceramic synthesis. A dihydroceramide desaturase introduces the 4,5-trans-double bond of sphingoceramide at the level of dihydroceramide. J. Biol. Chem. 272, 22432-22437.

Nakagawa, T., Kurose, T., Hino, T., Tanaka, K., Kawamukai, M., Niwa, Y., Toyooka, K., Matsuoka, K., Jinbo, T., and Kimura, T. (2007) Development of series of gateway binary vectors, pGWBs, for realizing efficient construction of fusion genes for plant transformation. J. Biosci. Bioeng. 104, 34-41.

Sperling, P., and Heinz, E. (2003) Plant sphingolipids: structural diversity, biosynthesis, first genes and functions. Biochem. Biophys. Acta 162, 1-15.

Sperling, P., Ternes, P., Moll, H., Franke, S., Zähringer, U., and Heinz, E. (2001) Functional characterization of sphingolipid C4-hydroxylase genes from Arabidopsis thaliana. FEBS Lett. 494, 90-94.

Sperling, P., Zähringer, U., and Heinz, E. (1998) A sphingolipid desaturase from higher plants. Identification of a new cytochrome $b_{5}$ fusion protein. J. Biol. Chem. 273, 2859028596.

Tamura, K., Mitsuhashi, N., Hara-Nishimura, I., and Imai, H. (2001) Characterization of an Arabidopsis cDNA encoding a subunit of serine palmitoyltransferase, the initial enzyme in sphingolipid biosynthesis. Plant Cell Physiol. 42, 12741281.

Ternes, P., Feussner, K., Werner, S., Lerche, J., Iven, T., Heilmann, I., Riezman, H., and Feussner, I. (2011) Disruption of the ceramide synthase LOH1 causes spontaneous cell death in Arabidopsis thaliana. New Phytol. 192, 841-854.

The Anigiosperm Phylogeny Group (2016) An update of the Angiosperm Phylogeny Group classification for the orders and families of flowering plants: APG IV. Bot. J. Linn. Soc. 181, 1-20.

Wright, B. S., Snow, J. W., O’Brien, T. C., and Lynch, D. V. (2003) Synthesis of 4-hydroxysphinganine and characterization of sphinganine hydroxylase activity in corn. Arch. Biochem. Biophys. 415, 184-192. 